\title{
ENGAGING END USERS IN AN AI-ENABLED SMART SERVICE DESIGN - THE APPLICATION OF THE SMART SERVICE BLUEPRINT SCAPE (SSBS) FRAMEWORK
}

\author{
Li, Fan; \\ Lu, Yuan \\ Eindhoven University of Technology
}

\begin{abstract}
Artificial Intelligence (AI) has expanded in a diverse context, it infiltrates our social lives and is a critical part of algorithmic decision-making. Adopting AI technology, especially AI-enabled design, by end users who are non-AI experts is still limited. The incomprehensible, untransparent decisionmaking and difficulty of using AI become obstacles which prevent these end users to adopt AI technology. How to design the user experience (UX) based on AI technologies is an interesting topic to explore.

This paper investigates how non-AI-expert end users can be engaged in the design process of an AIenabled application by using a framework called Smart Service Blueprint Scape (SSBS), which aims to establish a bridge between UX and AI systems by mapping and translating AI decisions based on UX. A Dutch mobility service called 'stUmobiel' was taken as a design case study. The goal is to design a reservation platform with stUmobiel end users. Co-creating with case users and assuring them to understand the decision-making and service provisional process of the AI-enabled design is crucial to promote users' adoption. Furthermore, the concern of AI ethics also arises in the design process and should be discussed in a broader sense.
\end{abstract}

Keywords: Artificial intelligence, Service design, Ethics, User experience, Smart service blueprint scape (SSBS) framework

\section{Contact:}

Li, Fan

Eindhoven University of Technology

Industrial design

Netherlands, The

f.li@tue.nl 


\section{INTRODUCTION}

In recent days, artificial intelligence (AI) and machine learning (ML) have been developed rapidly and have impacted almost all technical aspects of society. They can be applied in multiple fields such as autonomous vehicles and traffic management (Ye et al., 2018), health care (Panesar, 2019) and finance (Bahrammirzaee, 2010), which have all been enabled by algorithms as more and more functions have been ceded to algorithms (Sareen et al., 2020). AI algorithms are being used in computer science, data processing and autonomous decision-making. It is omnipresent in a human being's life by mediating and assisting the human decision-making process (Rai, 2020). In recent years, the availability of more data, the enhancement of computing power and the advancement of algorithms have promoted the revival of AI (Marr, 2019). Although algorithms can bring up highly personalized services and content, it is very difficult to explain their decisions and operations to users because of its' complexity (Gilpin et al., 2019). In particular, algorithmic phenomena raise issues such as fairness, accountability, transparency, and explainability (Shin, Zhong, \& Biocca, 2020). The black-box nature of the algorithmic process has led to focus on research in the explainability of AI, e.g., exploring the role of explainability and transparency features in adopting individualized recommendation services (Castelvecchi, 2016). Therefore, with the popularization of AI, the comprehension of algorithmic systems by end users have become an important problem that needs to be solved (Goodman and Flaxman, 2017). Correspondingly, not understanding the AI algorithms and their decision-making process is the barrier that prohibits non-AI-expert end users to adopt AI-enabled design. Hence, there is a need for AI to be comprehensible, transparent and trustworthy for these end users (Ribera and Lapedriza, 2019).

User experience (UX), which is a series of strategies for understanding users' needs and their behaviours which are then applied in the designing of useful, usable systems and services, can offer an associative approach to discover the relationship between AI and users (Chapman et al., 2016). In a sense, involving UX in the process of design with AI offers users transparency related to algorithmic decision-making, similarly, the AI algorithm can make predictions based on the user's needs and behaviours to create a new UX. Moreover, AI and UX have the same goals in that they are both designed to interpret user behaviour as well as predict what they will do next (Haughey, 2019). In the context of AI algorithms, services are driven and developed by growing data insights. Furthermore, these services also become more customized and accurate according to specific UX (Shin, Zhong, \& Biocca, 2020). For instance, at Amazon's website, the recommendation algorithms are used to provide personalized service for customers based on their interests and search history (Linden et al., 2003). YouTube uses algorithms to deal with collected user data in accordance with their duration of watching video clips, users' aggregated practices of sequential viewing, comments and matching keywords on title searching to recommend related personalized videos for the users (Airoldi et al., 2016). In order to make AI accessible and transparent to end users and the people directly affected by it, an important first step is to define UX.

To bridge the gap between the AI algorithm and UX for reaching effective transparency, the HCI community calls for multidisciplinary cooperation and a user-centric approach to explainability (Abdul et al., 2018; Wang et al., 2019). Recent work in this rising field tends to be based on the framework of human interpretation in the social sciences, and how explanatory features affect the user's interaction with AI (Liao et al., 2020; Wang et al., 2019) However, there is a lack of focus on embedding UX in designing with AI to make the design process transparent, explainable and comprehensible for those end users.

Therefore, this study aims to make the AI decision-making process transparent and comprehensible to end users. To realize this research goal, the authors applied the Smart Service Blueprint Scape (SSBS) framework as the design tool in designing an AI-enabled digital platform for end users in a Dutch mobility service called 'stUmobiel'. The purpose of using this framework is to demonstrate the relations between UX and AI technology in AI-enabled smart service design process. Co-creation was the research method that engages stUmobiel users in the design process of the smart platform in order to understand user needs and user behaviours for building up the relationship between UX and AI. Moreover, it also enabled the possibilities available to them to comprehend the tailored services and decisions of AI algorithms, and eventually increase the adoption of AI-enabled design. 


\section{LITERATURE REVIEW}

The academic literature of explainability and transparency of AI, engagement of end users and the related work of the SSBS framework in this study can be found in the literature review section. the three related literature works mentioned above can build up an overview of the academic outlook at the inability of understanding AI decision-making process. Additionally, applying the SSBS framework is an important approach to connect end users within the AI-enabled design process. Hence, the AI decision-making results will be transparent and understandable for non-professional end users.

\subsection{Explainability and transparency of Al}

The use of complex AI algorithms consisting of deep learning and other complex models, which aim to create black-box models, could lead to a lack of transparency and explainability (Carabantes, 2020). These transparency issues are also closely related to other classifiers, such as kernel machines, and decision trees. In which it is very difficult to interpret the algorithmic process of AI. (Lipton, 2018). The black-box models cannot explain how and why an AI-based system made decisions, meanwhile, users are just being provided with an answer directly without any explanation of why and how this answer come out (Bird et al., 2020). It is not easy to comprehend and make clear how an algorithm came to a particular decision, not just for a non-AI expert, sometimes even complex AI system developers cannot explain it specifically (Bathaee, 2018; Bird et al., 2020). Non-AI-expert end users have no idea how the data processing and algorithms lead to specific decisions. The problems associated with AI decision-making becoming a "black box" are observably exacerbated when treating ordinary end users who know little about how AI systems make decisions and still need to communicate with them. Therefore, explainability is essential when building trust, relation and connection with AI (Shin, 2020). Furthermore, questions like "What is it doing?" and "How is it doing that?" are always being faced as a user encounters recommended results from AI and algorithmic systems (Combs et al., 2020). Explainability in the AI algorithm decision-making process ensure the end users' confidence and trust, as well as the comprehension of why a system works in a particular way (Nicolaou et al., 2013; Samek and Müller, 2019).

\subsection{Engaging non-Al-expert end users in the design process}

The importance of co-creation and engagement with users is widely introduced and recommended in the human-centred design process. Engaging the non-AI-expert end users in the early planning phases or design process of the AI-enabled design can be better to understand user needs and ensure relevant safety, access, and utility are built in (Hoof, 2005). It has been recognized that effective technologies are those prioritizing the needs of users, acceptance of potential users, and appropriate prerequisites for adopting such technologies (Peek et al., 2016). Involving end users in the design process can expose undeveloped areas for improvement, thereby increasing user satisfaction and reducing barriers to adoption (Scandurra and Sjölinder, 2013). Co-creation can be an effective approach for better engaging the ordinary end users in the process of design with AI. It was defined as the process that customers or users take as a dominant role and co-create design values with developers together (Prahalad and Ramaswamy, 2004). Information and communication technologies (ICTs) offer the chance for users to engage in the innovation process of a product or service design. With the help of the Internet, companies and organizations can leverage the innovation potentials and knowledge of users (Di Gangi and Wasko, 2009). In order to generate new design ideas and formalize explainability for end users, most co-creation takes place in the innovation process of a service design, the conceptualization of a design concept, the explanation on how to measure the effect of explainability and the establishment of prototypes (Kohler et al., 2011; Samek et al., 2017). 


\subsection{Smart Service Blueprint Scape (SSBS) framework}

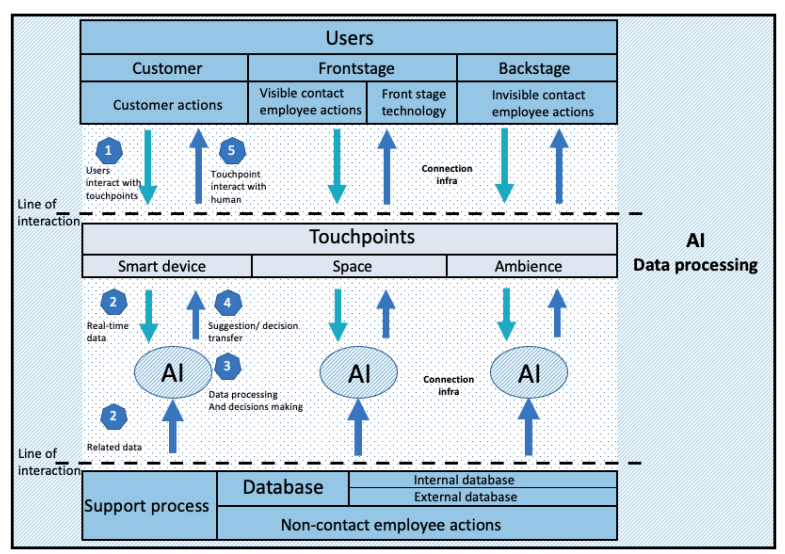

Figure 1. SSBS framework

The SSBS framework was created to design an AI-enabled smart service from UX perspective. It focuses on the process of how smart services achieve UX along with user needs and behaviours by interacting with AI data processing and database from the support process. It also provides the opportunity to bridge the gap between UX and AI technology (Li et al., 2020).

As can be seen from figure 1, it shows the main characteristics of the SSBS framework containing Users, Touchpoints, AI data processing and the Support process. Users include frontstage and backstage user actions, which represents the design process related to AI; touchpoints include a smart device, space and ambience, which are interactive roles between the user and the AI; support processes involve internal databases, external databases and non-contact employee actions, which are the backend of how the technology support department enables the delivery of the smart service. The interaction process has five steps, in step 1, users interact with a touchpoint to generate real-time interactive data. And then, in step 2, the generated data is transferred to the AI section for performing data processing. Meanwhile, the related data coming from the database is also sent to be processed with user interaction data for decision making. Steps 3 and 4 are the decision-making and transferring process. The data from the first two steps are processed by AI algorithms, after that decisions are made and transferred to touchpoints. In step 5 touchpoints receive the AI decisions and interact with a user engaging in the smart service process (Li et al., 2020).

In the current smart service design field, the design process between UX and AI technology are separate, which causes less connectivity between users and AI developers. Engineers strive to create an effective and reliable AI system for non-professional end users; however, such efforts can be wasted if these end users cannot interact with and comprehend the workings of the AI. The SSBS framework demonstrates the relationship between UX and AI, how user needs being transferred to the AI algorithms for decision making and eventually deliver matched user interfaces for creating new UX. The role of the designer is extended and includes being a coordinator which maintains the balance of the AI algorithmic process and UX which represents user needs and acceptance. The SSBS framework is also a tool that can guide designers to better understand the working process of AIenabled design and smooth communication among different stakeholders like users, service providers and technology experts in the service delivery process.

According to Li, Lu and Hands (2020), the SSBS framework will be a design approach which applies UX to inform AI technology. In the meanwhile, it could also serve as an inspiration for technologists to create meaningful UX through AI-enabled design i.e., smart service design.

\section{METHODOLOGY}

In this study, the SSBS framework was used as a tool for both data analysis and platform design. As it demonstrates the interactions between UX and AI, which also specifically describes how the user's needs and the AI algorithmic process reflect on each other to create new UX in terms of the user interface. At the same time, the authors used the Dutch mobility service stUmobiel as the case study. This mobility service provides a car driving service for senior adults to travel between their own houses and the elderly centre. stUmobiel involves seniors as end users, student drivers as frontstage employees and elderly centre managers as backstage employees. The authors co-created with these senior users (average age 65) to 
engage them in the design process of AI-enabled smart mobility service with four stages of the design process, i.e. Design insights for AI, Concept proposal and discussion and Prototype evaluation.

During the Design insights for AI phase, the authors participated in the mobility service delivery process of stUmobiel to examine the UX, then the author used the SSBS framework to analyse the current stUmobiel case to explore the opportunities for the AI technology embedment. Shadowing was used as an observation method for the authors to closely observe stUmobiel users' behaviour and UX in this service. It also helps to explore how the stUmobiel mobility service works for the senior users and how the other stakeholders like service managers and drivers are being involved in this service. After receiving enough data on the service delivery process of stUmobiel, the authors used the SSBS framework to illustrate the characteristics of user actions, frontstage and backstage employees, involved touchpoints and databases in this mobility service (refer to Figure 2). As a result, the design insights of AI in the current stUmobiel service can be concluded according to the SSBS analysis.

Based on the design insights for AI analysed by the SSBS framework, the authors proposed a design concept combined with UX and AI functionalities to design the stUmobiel smart platform. The design concept was then discussed among the authors and a co-creation group of four stUmobiel users. The users' needs and preferences of the UX in this concept was the key focus within the discussion. This section aimed to use co-creation method to engage senior users to reflect on the AI technology enabled UX and obtain their requirements on new UX for further AI functionalities' improvement.

Finally, the Computer-based Low-fidelity Prototype was made and used as the method for testing and evaluating the smart mobility service design. After organizing the user needs and service functions preference for the new design, the authors completed the concept within the SSBS framework based on the results of co-creation with senior adults together. A digital prototype showed to the four senior users and displayed the functionalities with a full user journey experience, which gave senior citizens a general overview of how this AI-enabled smart mobility service looks like. It was also animated on the screen to mimic its functionality and usage brought to four senior users for user testing and new a design experience.

\section{ETHICAL DECLARATION}

In order to let the senior citizens or their caregivers who will participate in the research know what their involvement in this research involves an ethics form which includes the research aim, introduction, proposed research methods, and intended participants were prepared. The participation was voluntary, and the shadowing method that using for joining the service delivery process all received agreement by senior users and their caregivers. To comply to General Data Protection Regulation (GDPR) and meet ethics and privacy considerations, the participants list is stored offline. Anonymized data is stored on the authors' university database and is only accessible to the involved researchers with password control.

\section{RESULts}

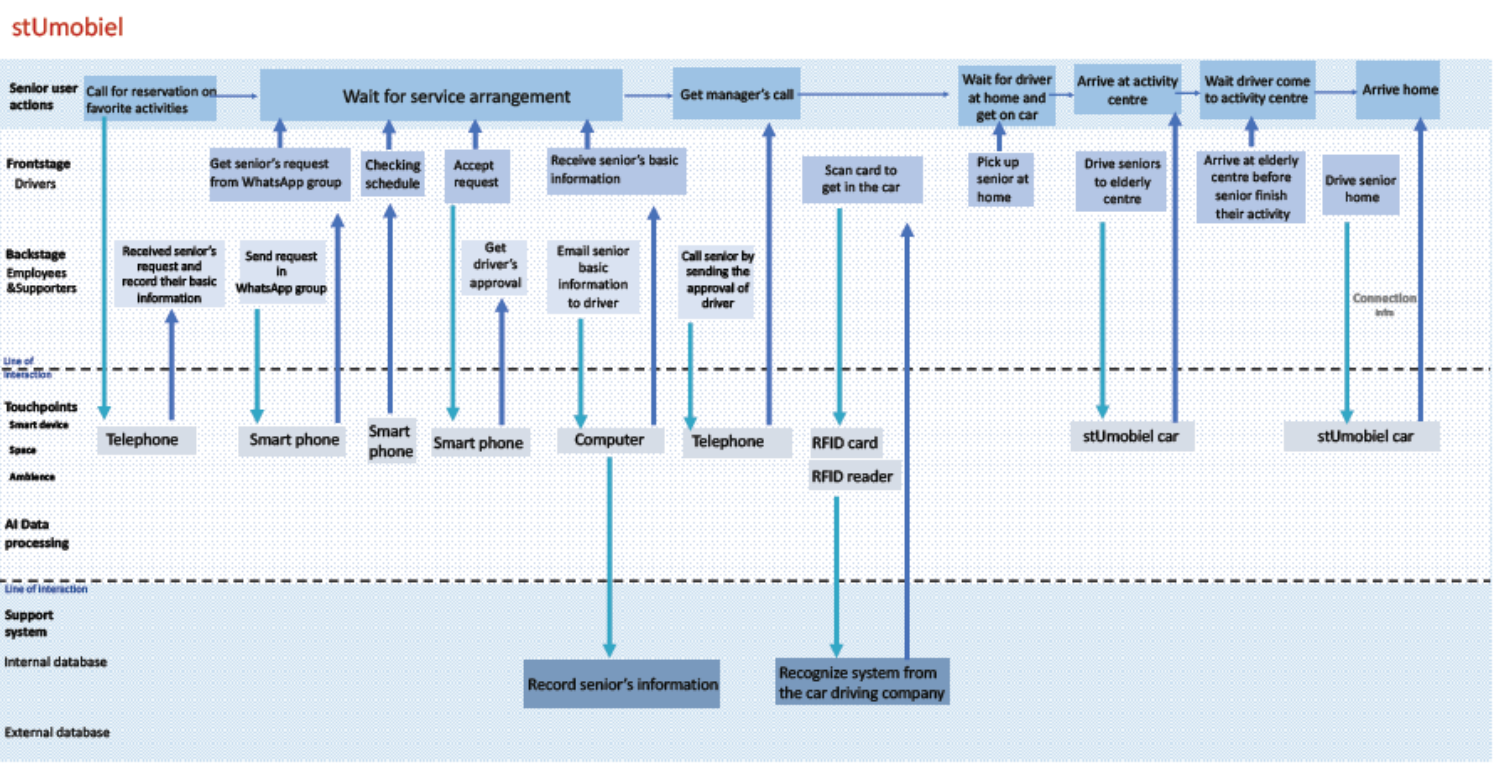

Figure 2. Service delivery process of stUmobiel 


\subsection{Design insights for Al: embedding $\mathrm{Al}$ and personalizing reservations}

As it shows in Figure 2, the service delivery process of stUmobiel was analysed by SSBS framework, the interactions among User actions, Front and backstage employees, Touchpoints and Database were defined in this figure. After the case analysis, two design insights were discovered: embedding AI technology in the reservation and the personalizing reservations on senior users' favourite activities.

The authors firstly found that for the stUmobiel senior users, making a phone call is the only way to reserve mobility service, which lacks digital and AI applications. After senior users sending their requests, the backstage employees need to check both older adults and drivers' agenda, then match them together to conduct a service. The whole reserving process was highly dependent on the backstage employees, who involves in not only record travel information of the senior users but also contact the frontstage drivers to get their feasible schedule. The WhatsApp group chatting is the only communication channel for stUmobiel staff to contact drivers. There is currently no direct way for these senior users to reserve their travel service without waiting for backstage employees' arrangement. Moreover, the dependence on backstage employees of the service is unsustainable for making stUmobiel smart and scalable. The platform enabled by AI Technology could be a design opportunity for updating the current stUmobiel service to a smart mobility service.

The second finding was that the personalized reservation for senior users is necessary. Because these users have strong preference on making appointments on their favourite activities. However, whenever they book stUmobiel services, they repeatedly reserve one or two favourite activities and ignore other options. Although the seniors' purpose is clear, they still need to rebook these favourite activities and sometimes the backstage employees cannot manage these reservations for seniors, as there might have some miscommunications and data loss when backstage employees was managing the appointments. There is a need to have a smart platform to deal with all the data and offer a personalized reservation for seniors to join their favourite activities.

Therefore, based on the two findings above, enabling AI technology in establishing a platform which can personalize reservations for end users is an opportunity for upgrading stUmobiel to a smart mobility service.

\subsection{Concept proposal and discussion: personalization and notification}

Based on the results of design insights for AI, the authors established an initial concept of a smart reservation platform by applying the SSBS framework for updating stUmobiel service. Four functions were focused on and summarized as: Profile page, Import your calendar, Reservations for yourself, and Reservation checklist. The basic information like their name, age, favourite activities, address and car preference should be both mentioned in the 'Profile page' and recorded in the user's smartphone and the backend database. In the 'Import your calendar' option, the senior users are asked to import their schedules stored in their phones, so that they can make reservations according to their agenda and stUmoiel smart platform can recommend suitable activities based on these data. The 'Reservation for yourself' option offers personalized reservation options that are relevant to their favourite activities. The 'Reservation checking list' aims to give an overview of the reserved mobility services in order to attend activities, which is also beneficial to the organization. Next, the authors brought this concept to a co-creation group consisting of four stUmobiel senior users. During the discussion, the senior users commented that they like the 'Reservation for you' option for personalized activities. As they prefer the options for reserving the activities corresponding to their favourite ones instead of selecting from all the activities that are available in the elderly centre. At the same time, they also expressed the requirement of getting notifications to recommend them the possibility to join their favourite activities when their schedule is blank.

According to the seniors' feedback, the authors updated the concept by adding the 'Notification messages' as the fifth function. Figure 3 demonstrates the updated concept based on the SSBS framework. Within this concept, the AI algorithms are enabled in two parts of the decision-making process. One part is making decisions on personalizing the reservations on users' favourite activities. Another is sending notification messages to notify users about coming activities which are customized according to the data on their agenda and booking history. When stUmobiel users book the travel services that are relevant to their favourite activities, this function is supported by the AI algorithm and the database. The internal database stores the user's favourite activities data and the external database stores available activities that seniors can attend in the elderly centre. The AI algorithm processes users' data on their favourite activities, and then drive patterns of these activities together 
with the data on available activities stored in the database of elderly centre. Consequently, the suggestions will be made and displayed through a smartphone user interface to support users to decide on attending suitable activities. The same algorithmic process also works on notifying the activities which fulfil users' preference, agenda and reservation history. Similarly, the AI algorithm can process the data of users' calendar, booking history and activities' timetable in elderly centre simultaneously to make predictions on the activities that they can potentially participate in. Afterwards, senior users will receive notification messages about their upcoming activities.

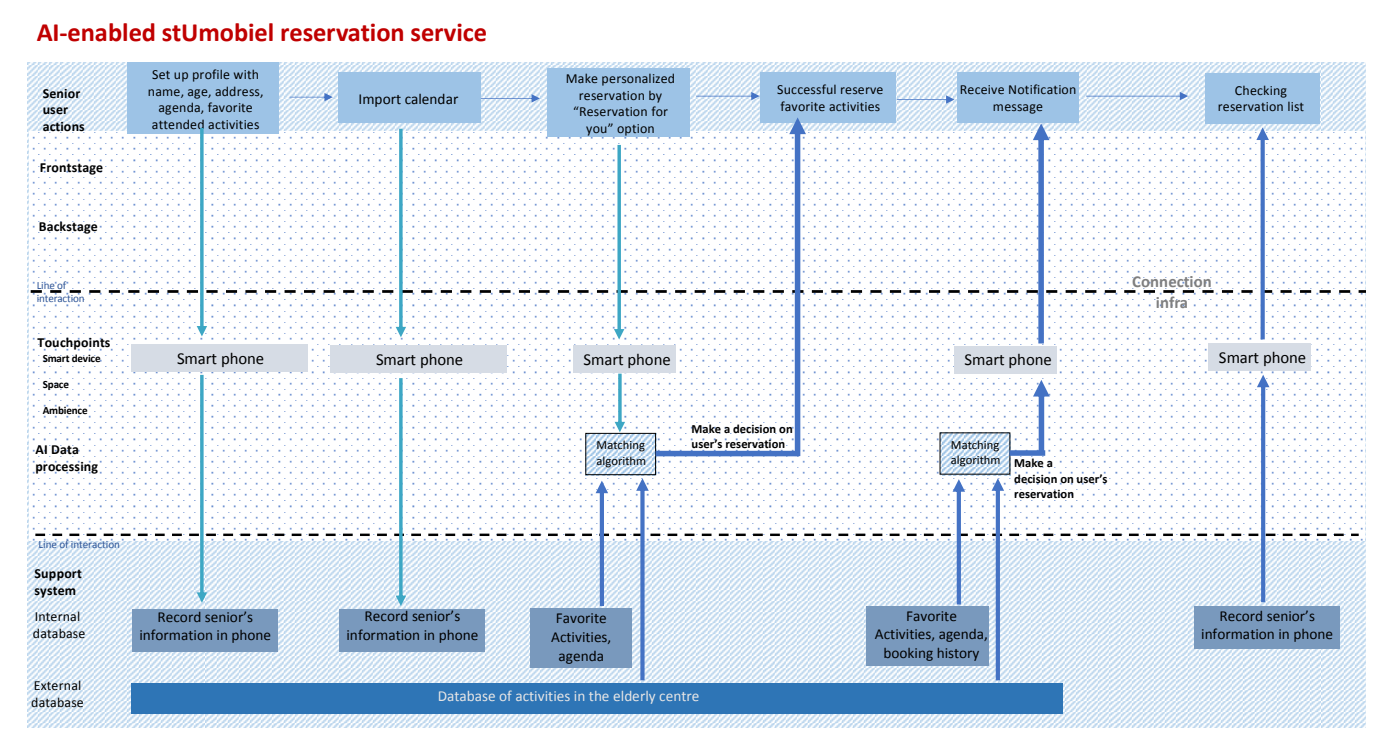

Figure 3. Concept analysed by SSBS framework

\subsection{Prototype evaluation: user interface, user context and user experience journey}

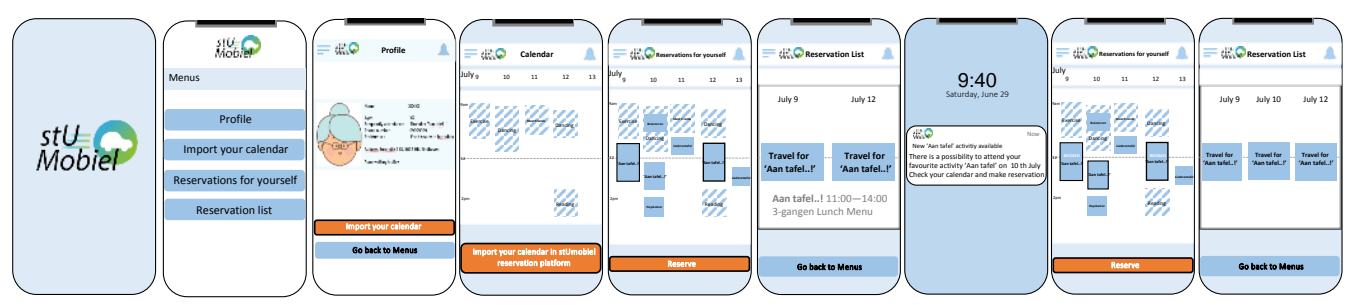

Figure 4. Low-fidelity software prototype

After conceptualizing the AI-enabled stUmobiel mobility service, the authors established a lowfidelity software prototype, which intends to simulate the functionalities and user interface of the smart mobility service. It also brought a general overview of the UX of reserving the travel service, receiving notification messages and organizing the reservation checklist. The prototype was tested from three parts: user interface, user context and user experience journey. After the user testing, the authors gathered feedback from the mentioned three parts.

In the user interface feedback, senior users were delighted by the clear Menu setting with only four options, clarifying the simple functions of profile, importing calendar, making reservations and checking reservation list. The fonts of the words are big and bold enough for them to use. The light blue colour base of the whole APP is highly relevant to the stUmobiel logo.

In the user context, the senior users mentioned that the timeslot of the activities was not clear, and description of the activities should be added more.

Through the whole user experience journey, stUmobiel users reflected that the using flow is smooth and it was easy to make reservation on the travel service for attending activities.

Additionally, the AI ethical concerns were risen up when senior users were evaluating the protocol, as they were concerned about the data protection of their personal information in the service platform. 


\section{DISCUSSION}

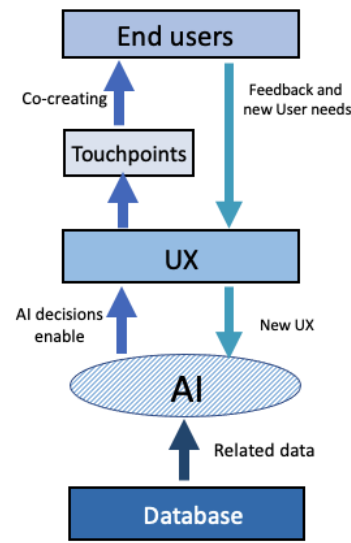

Figure 5. Co-creation in the Al-enabled design process

This study shows that the SSBS framework cannot only be used to demonstrate a correlation between UX and AI, but it can also be applied in the guiding of AI-enabled design.

The whole design process can be demonstrated in figure 5, which explains how the SSBS framework was applied in the AI-enabled design process of developing the stUmibiel smart mobility service. As can be seen from this figure, the design process was started from the AI aspect first, the SSBS framework was applied to analyse the current stUmobiel service for gaining insights into designing with AI.

Secondly, the concept with UX enabled by AI technology was brought to end users through a cocreation method. In the co-creation process, the authors and end users discussed and commented on the UX in the AI platform of the stUmobiel service, in the meanwhile, they also proposed further user needs to generate new UX.

Thirdly, the expected UX was translated to AI and at the same time, it also accepted the related data from the database for AI algorithms to processes the data. Afterwards, the related AI decisions were made for realizing the expected UX. Consequently, the touchpoints received the decisions and then transformed them as certain user interfaces to end users.

In this study, the co-creation method that empowers senior end users to engage in AI technology was discussed, specifically in the AI-enabled design process. It creates harmony between end users and AI technology to involve the user's needs and feedback to get new UX in the process of designing with AI. This makes the AI decision-making fulfil the UX and become explainable and transparent for end users.

It can also be explained in the stUmobiel concept discussion, the senior users co-created with the authors to discuss their preference and needs related to the concept. According to their comments, the senior end users expected to be notified about the coming activities that they would like to participate in based on their schedule and reservation history. To realize this UX, the data of senior users' schedule and using history was processed with AI algorithms and matched to the available activities they prefer to attend. Eventually, the end users receive a notification message to realize the UX in the form of user interface.

With the guidance of the SSBS framework, the designers can co-create with the end users to design with AI, help them understand how the AI influence UX and AI can also be influenced by UX. The whole design process with AI involved end users participating in. In the meanwhile, they expressed their perspectives on the UX enabled by AI and give suggestions to generate new UX. When the final UX delivered to them, they wouldn't get confused about the decisions made by the AI platform. They can understand AI decision-making, as they were co-creating with the designers together in the process of designing with AI.

Besides that, the user interface is seen as the channel to connect end users and designers. When designers design the UX and user interface based on AI, they need to connect the functionalities created by AI developers with the functions (including the interface), benefits and values that the end users will experience.

\section{CONCLUSION}

With the speedy growth of AI technology, it becomes important to approach AI-enabled design from not only the technology perspective, but also the UX point of view. However, the reality is that the 
incomprehensible and non-transparent decision-making of AI algorithms is the barriers that prevent nonAI-expert end users to understand and adopt AI technology. In accordance with the UX viewpoint, it would be significant and necessary to let these end users get to know the decision process in AI systems. Under this background, the authors selected stUmobiel, which provides travel service for senior users to attend activities, as the case study. Co-creation was adopted as the research method to engage stUmobiel users to take part in the design process. The authors and senior users explored opportunities together and applied the SSBS framework to design an AI-enabled smart platform for the stUmobiel service. The SSBS framework offered guidance for the designers to coordinate their actions with end users and AI algorithms for an explainable and transparent design. This closed the gap between normal end users and the AI system, as currently the AI technology and UX are fully separated.

In conclusion, this paper demonstrates how the SSBS framework can be applied to engage end users in the process of designing with AI and explore how UX and AI are influenced by each other. The end user participated in the co-creation process, learned about how the UX affected the AI technology, and how AI was influenced by UX. As a result, the design process can become transparent and understandable for the end user.

However, there is one aspect of great importance that has been left unexplored, which is the ethics of AI. The ethics of AI is an important issue, that is, how human developers, manufacturers and operators should act to minimize the ethical harm that AI may produce in society. AI is not only bringing benefits when people use it, it can also address ethical concerns. For instance, the risk of data protection and privacy, human rights, accountability, explainability and transparency. In this study, it was found that the stUmobiel users were worried about the safety of their personal information stored in the service platform during the prototype evaluation phase, which raised ethical concerns about data privacy and security in AI systems. Therefore, examining ethical principles in algorithms to make AI explainable and transparent is crucial, which can also support all the stakeholders in developing AIenabled design. Another aspect that can increase the researcher's focus is on design guidelines or regulations for designers to develop effective UX in AI systems.

All in all, based on these limitations, the focus on future research should be the exploration of the significance of ethical concerns within AI and its relationship to UX. Thereafter, certain design guidelines and ethical guidelines should be defined and combined with the SSBS framework to aid AI-enabled design.

\section{REFERENCES}

Abdul, A., Vermeulen, J., Wang, D., Lim, B.Y. and Kankanhalli, M. (2018), “Trends and trajectories for explainable, accountable and intelligible systems: An HCI research agenda", Conference on Human Factors in Computing Systems - Proceedings, available at: https://doi.org/10.1145/3173574.3174156.

Airoldi, M., Beraldo, D. and Gandini, A. (2016), "Follow the algorithm: An exploratory investigation of music on YouTube", Poetics, available at: https://doi.org/10.1016/j.poetic.2016.05.001.

Bahrammirzaee, A. (2010), "A comparative survey of artificial intelligence applications in finance: Artificial neural networks, expert system and hybrid intelligent systems", Neural Computing and Applications, available at: https://doi.org/10.1007/s00521-010-0362-z.

Bathaee, Y. (2018), “The Artificial Intelligence Black Box and the Failure of Intent and Causation”, Harvard Journal of Law \& Technology.

Bird, E., Fox-Skelly, J., Jenner, N., Larbey, R., Weitkamp, E. and Winfield, A. (2020), The Ethics of Artificial Intelligence: Issues and Initiatives, European Parliamentary Research Service.

Carabantes, M. (2020), "Black-box artificial intelligence: an epistemological and critical analysis", AI and Society, available at: https://doi.org/10.1007/s00146-019-00888-w.

Castelvecchi, D. (2016), “Can we open the black box of AI?”, Nature, available at: https://doi.org/10.1038/538020a.

Chapman, S., Fry, A., Deschenes, A. and McDonald, C.G. (2016), "Strategies to improve the user experience", Serials Review, available at: https://doi.org/10.1080/00987913.2016.1140614.

Combs, K., Fendley, M. and Bihl, T. (2020), “A Preliminary Look at Heuristic Analysis for Assessing Artificial Intelligence Explainability”, WSEAS transactions on computer research, available at: https://doi.org/10.37394/232018.2020.8.9.

Di Gangi, P.M. and Wasko, M. (2009), "The Co-Creation of Value: Exploring User Engagement in UserGenerated Content Websites", Sites the Journal Of 20Th Century Contemporary French Studies.

Gilpin, L.H., Bau, D., Yuan, B.Z., Bajwa, A., Specter, M. and Kagal, L. (2019), "Explaining explanations: An overview of interpretability of machine learning”, Proceedings - 2018 IEEE 5th International Conference on Data Science and Advanced Analytics, DSAA 2018, available at:

https://doi.org/10.1109/DSAA.2018.00018. 
Goodman, B. and Flaxman, S. (2017), "European union regulations on algorithmic decision making and a 'right to explanation"”, AI Magazine, available at: https://doi.org/10.1609/aimag.v38i3.2741.

Haughey, C. (2019), "How to Improve UX With AI and Machine Learning | Springboard Blog", available at: https://www.springboard.com/blog/improve-ux-with-ai-machine-learning/ (accessed 4 December 2020).

Hoof, J. Van. (2005), "Designing for older adults: principles and creative human factors approaches, by D. Fisk, A. Rogers, Neil Charness, Sara J. Czaja, Joseph Sharit; 2004”, Gerontechnology, available at: https://doi.org/10.4017/gt.2005.03.03.010.00.

Kohler, T., Fueller, J., Matzler, K. and Stieger, D. (2011), "CO-creation in virtual worlds: The design of the user experience”, MIS Quarterly: Management Information Systems, available at: https://doi.org/10.2307/23042808.

Liao, Q.V., Gruen, D. and Miller, S. (2020), “Questioning the AI: Informing Design Practices for Explainable AI User Experiences”, Conference on Human Factors in Computing Systems - Proceedings, available at: https://doi.org/10.1145/3313831.3376590.

Li, F., Lu, Y. and Hands, D. (2020), "Designing for an AI-enabled smart service adoption from a user experience perspective", Design Management Institute, pp. 3-7.

Linden, G., Smith, B. and York, J. (2003), “Amazon.com recommendations: Item-to-item collaborative filtering”, IEEE Internet Computing, available at: https://doi.org/10.1109/MIC.2003.1167344.

Lipton, Z.C. (2018), “The Mythos of Model Interpretability”, Queue, available at: https://doi.org/10.1145/3236386.3241340.

Marr, B. (2019), “Why Every Company Needs An Artificial Intelligence (AI) Strategy For 2019”, Forbes, available at: https://www.forbes.com/sites/bernardmarr/2019/03/21/why-every-company-needs-anartificial-intelligence-ai-strategy-for-2019/?sh=4a62e03e68ea (accessed 18 November 2020).

Nicolaou, A.I., Ibrahim, M. and Van Heck, E. (2013), "Information quality, trust, and risk perceptions in electronic data exchanges", Decision Support Systems, available at:https: //doi.org/10.1016/j.dss.2012.10.024.

Panesar, A. (2019), Machine Learning and AI for Healthcare, Machine Learning and AI for Healthcare, available at: https://doi.org/10.1007/978-1-4842-3799-1.

Peek, S.T.M., Wouters, E.J.M., Luijkx, K.G. and Vrijhoef, H.J.M. (2016), "What it Takes to successfully implement technology for aging in place: Focus groups with stakeholders", Journal of Medical Internet Research, available at: https://doi.org/10.2196/jmir.5253.

Prahalad, C.K. and Ramaswamy, V. (2004), "Co-creating unique value with customers", Strategy \& Leadership, available at: https://doi.org/10.1108/10878570410699249.

Rai, A. (2020), "Explainable AI: from black box to glass box", Journal of the Academy of Marketing Science, available at: https://doi.org/10.1007/s11747-019-00710-5.

Ribera, M. and Lapedriza, A. (2019), "Can we do better explanations? A proposal of user-centered explainable AI", CEUR Workshop Proceedings.

Samek, W. and Müller, K.R. (2019), “Towards Explainable Artificial Intelligence”, Lecture Notes in Computer Science (Including Subseries Lecture Notes in Artificial Intelligence and Lecture Notes in Bioinformatics), available at: https://doi.org/10.1007/978-3-030-28954-6_1.

Samek, W., Wiegand, T. and Müller, K.R. (2017), "Explainable artificial intelligence: Understanding, visualizing and interpreting deep learning models", ArXiv.

Sareen, S., Saltelli, A. and Rommetveit, K. (2020), "Ethics of quantification: illumination, obfuscation and performative legitimation”, Palgrave Communications, available at: https://doi.org/10.1057/s41599-0200396-5.

Scandurra, I. and Sjölinder, M. (2013), "Participatory design with seniors: Design of future services and iterative refinements of interactive eHealth services for old citizens", Journal of Medical Internet Research, available at: https://doi.org/10.2196/med20.2729.

Shin, D., Zhong, B. and Biocca, F.A. (2020), "Beyond user experience: What constitutes algorithmic experiences?”, International Journal of Information Management, Elsevier Ltd, Vol. 52, p. 102061.

Shin, F.P.D. (2020), "The Effects of Explainability and Causability on Perception, Trust, and Acceptance: Implications for Explainable AI", International Journal of Human-Computer Studies, available at: https://doi.org/10.1016/j.ijhcs.2020.102551.

Wang, D., Yang, Q., Abdul, A. and Lim, B.Y. (2019), "Designing theory-driven user-centric explainable AI”, Conference on Human Factors in Computing Systems - Proceedings, available at: https://doi.org/10.1145/3290605.3300831.

Ye, H., Liang, L., Li, G.Y., Kim, J., Lu, L. and Wu, M. (2018), "Machine Learning for Vehicular Networks: Recent Advances and Application Examples", IEEE Vehicular Technology Magazine, available at: https://doi.org/10.1109/MVT.2018.2811185. 